\title{
MARTINGALE APPROACH TO PRICING PERPETUAL AMERICAN OPTIONS
}

\author{
By Hans U. Gerber \\ Université de Lausanne, Switzerland \\ ANd Elias S.W. ShIU \\ The University of Iowa, U.S.A.
}

\begin{abstract}
The method of Esscher transforms is a tool for valuing options on a stock, if the logarithm of the stock price is governed by a stochastic process with stationary and independent increments. The price of a derivative security is calculated as the expectation, with respect to the risk-neutral Esscher measure, of the discounted payoffs. Applying the optional sampling theorem we derive a simple, yet general formula for the price of a perpetual American put option on a stock whose downward movements are skip-free. Similarly, we obtain a formula for the price of a perpetual American call option on a stock whose upward movements are skip-free. Under the classical assumption that the stock price is a geometric Brownian motion, the general perpetual American contingent claim is analysed, and formulas for the perpetual down-and-out call option and Russian option are obtained. The martingale approach avoids the use of differential equations and provides additional insight. We also explain the relationship between Samuelson's high contact condition and the first order condition for optimality.
\end{abstract}

\section{KEYWORDS}

Black-Scholes formula; option-pricing theory; equivalent martingale measure; Esscher transform; perpetual American call option; perpetual American put option; perpetual down-and-out American call option; perpetual American strangle; perpetual American straddle; Russian option; optional sampling theorem; optimal stopping; high contact condition; smooth pasting condition.

\section{INTRODUCTION}

The option-pricing theory of BLACK and SCHOLES (1973) is perhaps the most important development in the theory of financial economics in the past two decades. A fundamental insight in advancing the theory is the concept of risk-neutral valuation introduced by Cox and Ross (1976). Further elaboration on this idea was given by HARRISON and KREPS (1979), HARRISON and PLISKA (1981) and others 
under the terminology of equivalent martingale measure. It is now understood that the absence of arbitrage is "essentially" equivalent to the existence of an equivalent martingale measure, and some authors (DYBVIG and Ross, 1987; SCHACHermayer, 1992) call this the Fundamental Theorem of Asset Pricing.

Under the assumption that the logarithm of the stock price is governed by a stochastic process with stationary and independent increments, one may determine such an equivalent martingale measure by a time-honored technique in actuarial science - the Esscher transform (EsSCHER, 1932). An Esscher transform induces an equivalent probability measure on such a stock-price process. The risk-neutral Esscher parameter (which is unique) is determined so that the stock price, discounted by the risk-free interest rate less the dividend yield, becomes a martingale under the new probability measure. The price of a derivative security is the supremum of the expected discounted payoffs, where the expectation is taken with respect to this equivalent martingale measure and the discounting is calculated using the risk-free interest rate.

The pricing of American options with a finite expiration date has been a challenging problem in the field of financial economics. A main difficulty is the determination of the optimal exercise boundary. Some papers on American options in the past decade are Bensoussan (1984), MACMILLAN (1986), BARONE-AdESI and Whaley (1987), OMBERG (1987), KARATZAS (1988), JaILlET, LAMBERTON and LAPEYRE (1990), KIM (1990), JACKA (1991), CARR, JARROW and MYNENI (1992), Myneni (1992), Chesney, Elliot and Gibson (1993), Lamberton (1993), Hull and White (1993), and TILleY (1993). In this paper we study the pricing of American options without expiration date by means of the Esscher transform and the optional sampling (stopping) theorem. This is a more tractable problem because the optimal exercise boundary of a perpetual American option does not vary with respect to the time variable. We derive a simple, yet general formula for the price of a perpetual American put option on a stock whose downward movements are skip-free (jump-free). Similarly, we obtain a formula for the price of a perpetual American call option on a stock whose upward movements are skip-free. In the appendix, we present a family of stochastic processes for modeling such stock-price movements. This family includes the Wiener process, gamma process and inverse Gaussian process, and combinations of such processes.

Under the classical assumption that the stock price is a geometric Brownian motion, the general perpetual American contingent claim is analysed, and formulas for the perpetual down-and-out call option and Russian option are obtained. The martingale approach avoids the use of differential equations and provides additional insight. We also explain the relationship between Samuelson's high contact condition and the first order conditions for optimality.

\section{THE RISK-NEUTRAL ESSCHER TRANSFORM}

Let $S(t)$ be the price of a stock at time $t, t \geq 0$. We assume that the process, $\{X(t)\}_{t \geq 0}$, defined by

$$
S(t)=S(0) e^{X(t)}, \quad t \geq 0,
$$


is one with stationary and independent increments. Let

$$
F(x, t)=\operatorname{Pr}[X(t) \leq x], \quad t \geq 0,
$$

be the distribution of the random variable $X(t)$, and

$$
M(z, t)=E\left[e^{z X(t)}\right], \quad t \geq 0,
$$

its moment generating function. Under a mild continuity condition (BREIMAN, 1968, Section 14.4),

$$
M(z, t)=[M(z, 1)]^{t}, \quad t \geq 0 .
$$

While the Esscher transform of a random variable is a well-established concept, in this paper we consider the Esscher transform of a stochastic process which satisfies (2.4). The Esscher transform (parameter $h$ ) of $\{X(t)\}_{t \geq 0}$ is again a process with stationary and independent increments; the modified distribution of $X(t)$ is now

$$
\begin{aligned}
F(x, t ; h) & =\operatorname{Pr}[X(t) \leq x ; h] \\
& =\frac{\int_{-\infty}^{x} e^{h y} d F(y, t)}{\int_{-\infty}^{\infty} e^{h y} d F(y, t)} \\
& =\frac{1}{M(h, t)} \int_{-\infty}^{x} e^{h y} d F(y, t) .
\end{aligned}
$$

The corresponding moment generating function is

$$
M(z, t ; h)=\frac{M(z+h, t)}{M(h, t)} .
$$

It follows from (2.4) that

$$
\begin{aligned}
M(z, t ; h) & =\left[\frac{M(z+h, 1)}{M(h, 1)}\right]^{t} \\
& =[M(z, 1 ; h)]^{t} .
\end{aligned}
$$

Because the exponential function is positive, the old and new measures have the same null sets, i.e., they are equivalent probability measures. The appropriate parameter $h=h^{*}$ is determined according to the principle of risk-neutral valuation (Cox and Ross, 1976), or, using the terminology of HARRISON and KrEPS (1979) and HARRISON and PliskA (1981), we seek $h=h^{*}$ to obtain an equivalent martingale measure.

In this paper we assume that the risk-free force of interest is constant, and it is denoted by $\delta$. We also assume that the market is frictionless and trading is 
continuous. There are no taxes, no transaction costs, and no restriction on borrowing or short sales. All securities are perfectly divisible. Furthermore, we assume that the stock pays a continuous stream of dividends, at a rate proportional to its price, i.e., there is a nonnegative constant $\rho$ such that the dividend paid between time $t$ and $t+d t$ is $S(t) \rho d t$. The parameter $h=h^{*}$ is chosen so that the process $\left\{e^{-(\delta-\rho) t} S(t)\right\}_{t \geq 0}$ is a martingale with respect to the probability measure corresponding to $h^{*}$. In particular,

$$
S(0)=E\left[e^{-(\delta-\rho) t} S(t) ; h^{*}\right]
$$

hence, by (2.1) and (2.6),

$$
\begin{aligned}
e^{(\delta-\rho) t} & =E\left[e^{X(t)} ; h^{*}\right] \\
& =\left[M\left(1,1 ; h^{*}\right)\right]^{t},
\end{aligned}
$$

or

$$
\ln \left[M\left(1,1 ; h^{*}\right)\right]=\delta-\rho .
$$

The Esscher measure corresponding to the parameter $h^{*}$ is called the risk-neutral Esscher measure. The price of a derivative security, whose payments depend on $\{S(t)\}$, is calculated as a discounted expected value, where the expectation is taken with respect to the risk-neutral Esscher measure.

Under some regularity conditions, equation (2.8) has a unique solution. To see this, consider the function

$$
g(h)=\ln [M(1,1 ; h)]=\ln [M(1+h, 1)]-\ln [M(h, 1)] .
$$

The formula

$$
\frac{d}{d h} E[X(1) ; h]=\operatorname{Var}[X(1) ; h]
$$

shows that $E[X(1) ; h]$ is an increasing function in $h$. Hence

$$
g^{\prime}(h)=E[X(1) ; 1+h]-E[X(1) ; h]
$$

is positive, showing that $g(h)$ is an increasing function. This proves the uniquencess of the solution of equation (2.8), which is

$$
g(h)=\delta-\rho .
$$

To discuss the existence of the solution, let $M$ and $m$ denote the right and left end point of the (essential) range of $X(1)$, respectively. (M may be $+\infty$ and $m$ may be $-\infty)$. We may assume

$$
m+\rho<\delta<M+\rho
$$

or

$$
m<\delta-\rho<M
$$


because otherwise arbitrage would be possible. Let $(a, b)$ denote the interval of values of $h$ for which $g(h)$ exists. Under some regularity conditions,

$$
\lim _{h \downarrow a} g(h)=m, \quad \lim _{h \uparrow b} g(h)=M,
$$

in which case (2.8) does have a solution. It should be noted that, although the risk-neutral Esscher measure is unique, there may be other equivalent martingale measures; see Delbaen and HAEzEndonck (1989) for a study on equivalent martingale measures of compound Poisson processes.

The price of a derivative security is taken as the expectation of its discounted payoffs with respect to the risk-neutral Esscher measure. For example, consider a European call option on the stock with exercise price $K$ and exercise date $t, t>0$. Let $I(\cdot)$ denote the indicator function and $k=\ln [K / S(0)]$. The price of the option (at time 0 ) is

$$
\begin{aligned}
& e^{-\delta t} E\left[(S(t)-K) I(S(t)>K) ; h^{*}\right] \\
& =e^{-\delta t} E\left[S(t) I(S(t)>K) ; h^{*}\right]-e^{-\delta t} K E\left[I(S(t)>K) ; h^{*}\right]
\end{aligned}
$$

The second expectation in the right-hand side of (2.9) is

$$
\operatorname{Pr}\left[S(t)>K ; h^{*}\right]=1-F\left(\kappa, t ; h^{*}\right) .
$$

To evaluate the first expectation in the right-hand side of (2.9), note that, for each measurable function $g(\cdot)$,

$$
\begin{aligned}
E[g(S(t)) ; h] & =\frac{E\left[g(S(t)) e^{h X(t)}\right]}{E\left[e^{h X(t)}\right]} \\
& =\frac{E\left[g(S(t)) S(t)^{h}\right]}{E\left[S(t)^{h}\right]} .
\end{aligned}
$$

With this formula, the following result can be proved.

Lemma : Let $h$ and $k$ be two real numbers. Assume that the Esscher transforms of parameters $h$ and $h+k$ exist. Then, for each measurable function $\psi(\cdot)$,

$$
E\left[S(t)^{k} \psi(S(t)) ; h\right]=E\left[S(t)^{k} ; h\right] E[\psi(S(t)) ; h+k] .
$$

Applying the Lemma [with $k=1, \psi(x)=I(x>K)$ and $\left.h=h^{*}\right]$ and (2.7), we obtain

$$
\begin{aligned}
E\left[S(t) I(S(t)>K) ; h^{*}\right] & =E\left[S(t) ; h^{*}\right] E\left[I(S(t)>K) ; h^{*}+1\right] \\
& =S(0) e^{(\delta-\rho) t} \operatorname{Pr}\left[(S(t)>K) ; h^{*}+1\right] .
\end{aligned}
$$

Thus the price of the European call option is

$$
S(0) e^{-\rho t}\left[1-F\left(\kappa, t ; h^{*}+1\right)\right]-K e^{-\delta t}\left[1-F\left(\kappa, t ; h^{*}\right)\right] .
$$


If $\{X(t)\}$ is a Wiener process with variance per unit time $\sigma^{2}$, then (2.12) (with (4.2) below) yields the expression

$$
S(0) e^{-\rho t} \Phi\left(\frac{-\kappa+\left(\delta-\rho+\sigma^{2} / 2\right) t}{\sigma \sqrt{t}}\right)-K e^{-\delta t} \Phi\left(\frac{-K+\left(\delta-\rho-\sigma^{2} / 2\right) t}{\sigma \sqrt{t}}\right),
$$

where $\Phi(\cdot)$ denotes the standardized normal distribution. For $\rho=0$ this is the celebrated Black-Scholes formula. Formula (2.13) is the same as formula (53) in SMITH (1976).

\section{Remarks :}

(1) We assume that the stock pays dividends at a constant proportional rate $\rho$. If all dividends are reinvested in the stock, then each share of the stock at time 0 grows to $e^{\rho t}$ shares at time $t$; this gives an interpretation for formula (2.7),

$$
S(0)=E\left[e^{-\delta t} S(t) e^{\rho t} ; h^{*}\right] .
$$

On the other hand, we can also consider the situation where none of the dividends are reinvested in the stock, leading to the intuitive formula:

$$
S(0)=E\left[\int_{0}^{t} e^{-\delta u} S(u) \rho d u+e^{-\delta t} S(t) ; h^{*}\right] .
$$

To prove (2.14), we interchange the order of expectation and integration on the right-hand side and apply the formula

$$
E\left[e^{-\delta u} S(u) ; h^{*}\right]=e^{-\rho u} S(0)
$$

thus

$$
\begin{aligned}
\text { R.H.S. } & =S(0)\left(\int_{0}^{t} e^{-\rho u} \rho d u+e^{-\rho r}\right) \\
& =S(0) \\
& =\text { L.H.S. }
\end{aligned}
$$

(2) Formula (2.12) may be used to price currency exchange options, with $S(t)$ denoting the spot exchange rate at time $t, \delta$ the domestic force of interest and $\rho$ the foreign force of interest. In this context, (2.13) is known as the Garman-Kohlhagen formula.

\section{PRICING PERPETUAL AMERICAN OPTIONS}

In this section, by applying the optimal sampling theorem, we derive pricing formulas for perpetual American put and call options on a stock. We make the assumptions about stock prices and dividends that were introduced in the previous section. In addition, when pricing a perpetual American put option, we assume that 
the downward movements of the stock price are skip-free. Similarly, when pricing a perpetual American call option, we assume that the upward movements of the stock price are skip-free. Under these convenient assumptions, attractive formulas can be obtained.

First, we consider a perpetual American put option with exercise price $K$. We temporarily assume that $K<S(0)$, so that an immediate exercise of the option can be excluded. The owner of this option exercises it according to some strategy: a stopping time $T$. Then, at time $T$, he will get

$$
(K-S(T))_{+},
$$

where $x_{+}=\operatorname{Max}(x, 0)$. Thus the value (at time 0 ) associated with the strategy is

$$
E\left[e^{-\delta T}(K-S(T))_{+} ; h^{*}\right] .
$$

To maximize this expression, we can limit ourselves to stationary strategies of the form

$$
T_{L}=\inf \{t \mid S(t) \leq L\},
$$

where $L \leq K$; the option is exercised the first time when (if ever) the price of the stock falls below or equals the level $L$. The price of the option is the maximal value of

$$
E\left[e^{-\partial T_{L}}\left(K-S\left(T_{L}\right)\right)_{+} ; h^{*}\right] .
$$

With the assumption that the stock-price process, $\{S(t)\}_{t \geq 0}$, is skip-free downwards, the stock price is equal to $L$ at the time when the option is exercised, i.e.,

$$
L=S\left(T_{L}\right)=S(0) e^{X\left(T_{L}\right)} .
$$

For simplicity, denote the current stock price $S(0)$ by $S$ and expression (3.3) by $V(S, L)$. Since $L \leq K$,

$$
V(S, L)=(K-L) E\left[e^{-\delta T_{L}} ; h^{*}\right] .
$$

The expectation in (3.5) is a Laplace transform of $T_{L}$ and can be calculated by the following classical argument.

Consider the stochastic process $\left\{e^{-\partial t+\theta X(t)}\right\}_{t \geq 0}$. For $t \leq T_{L}$, it is a bounded martingale with respect to the risk-neutral Esscher measure if the coefficient $\theta$ is the negative solution of the equation

$$
E\left[e^{-\delta t+\theta X(t)} ; h^{*}\right]=1
$$

or

$$
M\left(\theta, 1 ; h^{*}\right)=e^{\delta} .
$$

Equation (3.6) has two (real) solutions; one is negative and the other is greater than one. To see this, consider the function

$$
\phi(\theta)=M\left(\theta, 1 ; h^{*}\right)=E\left[e^{\theta X(1)} ; h^{*}\right] .
$$


Since

$$
\phi^{\prime \prime}(\theta)=E\left[X(1)^{2} e^{\theta X(1)} ; h^{*}\right]>0,
$$

the function $\phi(\theta)$ is convex. Consequently, equation (3.6),

$$
\phi(\theta)=e^{\delta}
$$

has at most two solutions. We note that

$$
\phi(0)=1<e^{\delta}
$$

and, because of (2.8),

$$
\phi(1)=e^{\delta-\rho}<e^{\partial}
$$

Let us assume that

$$
\operatorname{Pr}[X(1)<0]>0
$$

and

$$
\operatorname{Pr}[X(1)>0]>0,
$$

from which it follows that $\phi(\theta) \rightarrow+\infty$ for $\theta \rightarrow-\infty$ and for $\theta \rightarrow+\infty$. Thus equation (3.6) has two solutions, $\theta_{0}<0$ and $\theta_{1}>1$.

By the optional sampling theorem, we have

$$
E\left[e^{-\delta T_{L}+\theta_{i} X\left(T_{l}\right)} ; h^{*}\right]=1,
$$

which, because of (3.4), becomes

$$
E\left[e^{-\delta T_{L}} ; h^{*}\right]=\left(\frac{L}{S}\right)^{-\theta_{0}} .
$$

Applying (3.7) to (3.5) yields, for $S \geq L$ and $K>L$,

$$
V(S, L)=(K-L)\left(\frac{L}{S}\right)^{-\theta_{11}} .
$$

For a given current stock price $S$, we seek the maximal value of (3.8) by varying the option-exercise boundary $L$. Let $V_{L}$ denote the partial derivative of $V$ with respect to $L$. Solving the equation

$$
V_{L}(S, L)=0
$$

yields the optimal exercise boundary

$$
L=\tilde{L}=\frac{-\theta_{0}}{1-\theta_{0}} K .
$$

Thus the maximal value is

$$
V(S, \tilde{L})=\frac{K}{1-\theta_{0}}\left[\frac{-K \theta_{0}}{S\left(1-\theta_{0}\right)}\right]^{-\theta_{0}} .
$$


This is the price of the perpetual American put option provided that $S \geq \tilde{L}$. For $S<\tilde{L}$, the option is exercised immediately and the price is simply $K-S$. Hence the option price is

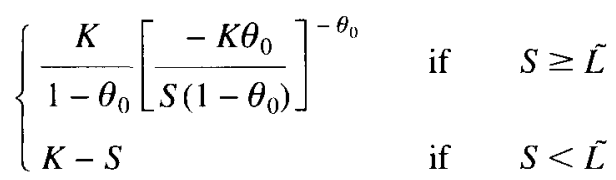

It may seem surprising that $\delta$ and $\rho$ do not appear in (3.10). However, they were used to determine $\theta_{0}$.

Next we study the pricing of a perpetual American call option with exercise price $K$, and we temporarily assume that $K>S$. For $M \geq K$, let

$$
T_{M}=\inf \{t \mid S(t) \geq M\}
$$

and

$$
W(S, M)=E\left[e^{-\delta T_{M}}\left(S\left(T_{M}\right)-K\right)_{+} ; h^{*}\right] .
$$

With the assumption that the stock-price process, $\{S(t)\}_{t \geq 0}$, is skip-free upwards, the stock price is equal to $M$ at the time when the option is exercised, i.e., $S\left(T_{M}\right)=M$. Since $M \geq K$, formula (3.12) becomes

$$
W(S, M)=(M-K) E\left[e^{-\delta T_{M}} ; h^{*}\right] .
$$

The expectation in (3.13) is evaluated in the same way as above, except that we now use $\theta_{1}$, the positive root of (3.6), to make sure that $\left\{\exp \left[-\delta t+\theta_{1} X(t)\right]\right\}$ is a bounded martingale (with respect to the risk-neutral Esscher measure) for $t \leq T_{M}$. The resulting formula is

$$
E\left[e^{-\delta T_{M}} ; h^{*}\right]=\left(\frac{S}{M}\right)^{\theta_{1}} .
$$

For given current stock price $S$, the maximal value of

$$
W(S, M)=(M-K)\left(\frac{S}{M}\right)^{\theta_{1}}
$$

is attained at

$$
M=\tilde{M}=\frac{\theta_{1}}{\theta_{1}-1} K,
$$

and

$$
W(S, \tilde{M})=\frac{K}{\theta_{1}-1}\left[\frac{S\left(\theta_{1}-1\right)}{K \theta_{1}}\right]^{\theta_{1}} .
$$

This gives the price of the perpetual American call option provided $S \leq \tilde{M}$. For $S>\tilde{M}$, the option is exercised immediately and the price is simply $S-K$. Thus the 
option price is

$$
\left\{\begin{array}{lll}
\frac{K}{\theta_{1}-1}\left[\frac{S\left(\theta_{1}-1\right)}{K \theta_{1}}\right]^{\theta_{1}} & \text { if } & S \leq \tilde{M} \\
S-K & \text { if } & S>\tilde{M} .
\end{array}\right.
$$

Remarks : As the dividend yield $\rho$ tends to 0 , the coefficient $\theta_{1}$ tends to 1 , the optimal exercise boundary $\tilde{M}$ tends to $\infty$, and the price of the perpetual American call option tends to $S$, the current stock price. These limiting results can be verified by direct calculations: for $\rho=0, \theta_{1}=1,(3.15)$ reduces to

$$
W(S, M)=\left(1-\frac{K}{M}\right) S, \quad M \geq K .
$$

Since this is a strictly increasing function of $M$, its supremum is not attained for a finite value of $M$, and the maximal value (the value of the option) is $S$. Thus, if $\rho=0$, the perpetual American call option will never be exercised, but nevertheless it has a positive value. To avoid this anomaly (to which INGERSOLL (1987, p. 373) refers as the problem of "infinities") we might modify the payoff of the call option as

$$
\left[(S(T)-K)_{+}\right]^{\alpha}, \quad 0<\alpha<1
$$

Then

$$
W(S, M)=(M-K)^{\alpha} \frac{S}{M},
$$

which is maximal for

$$
\tilde{M}=\frac{K}{1-\alpha} .
$$

\subsection{The high contact condition}

Each of (3.10) and (3.18), as a function of the current stock price $S$, has a continuous first derivative, because

$$
\begin{aligned}
& V(\tilde{L}, \tilde{L})=K-\tilde{L}, \\
& V_{S}(\tilde{L}, \tilde{L})=-1, \\
& W(\tilde{M}, \tilde{M})=\tilde{M}-K,
\end{aligned}
$$

and

$$
W_{S}(\tilde{M}, \tilde{M})=1 .
$$

Formulas (3.1.1) and (3.1.2) are special cases of the so-called high contact condition (SAMUELSON, 1965); in the literature about optimal stopping problems (ShIRAYAYEV, 1978, p. 160) the term is smooth pasting condition. SHEPP and 
ShIRYAEV (1983) use the term the principle of smooth fit and attribute it to Kolmogorov. (Shirayayev is the same person as Shiryaev).

MERTON (1973, p. 171, footnote $60 ; 1990$, p. 296, footnote 47 ) has derived the high contact condition as a first order condition necessary for optimality. (Merton's proof is reformulated on page 189 of BREKKE and ØKSENDAL (1991)). Under some weak conditions, the converse is also true - a solution proposal to an optimal stopping problem satisfying the high contact condition is in fact an optimal solution to the problem; a recent paper on this is BREKKE and ØKSENDAL (1991). It is easy to check that condition (3.1.1) does determine the optimal exercise boundary $\tilde{L}$, while (3.1.2) determines $\tilde{M}$.

We now derive a formula explaining how the high contact condition (3.1.1) and optimality for $V(S, \cdot)$ are related. Let

$$
\lambda(S, L)=E\left[e^{-\delta T_{L}} ; h^{*}\right] .
$$

From (3.7) or simply by interpretation, it follows that, for $0<x<S-L$,

$$
\lambda(S, L)=\lambda(S, L+x) \lambda(L+x, L)
$$

(cf. Lemma 7.1 on page 243 of KARLIN and TAYLOR (1981)). Differentiating (3.1.4) with respect to $x$ and setting $x=0$ yields

$$
0=\lambda_{L}(S, L)+\lambda(S, L) \lambda_{S}(L, L) .
$$

Now, let

$$
\pi(x)=(K-x)_{+}
$$

denote the payoff function. Then (3.5) becomes

$$
V(S, L)=\pi(L) \lambda(S, L) .
$$

Differentiating (3.1.7) with respect to $L$ and applying (3.1.5) yields

$$
\begin{aligned}
V_{L}(S, L) & =\pi^{\prime}(L) \lambda(S, L)+\pi(L) \lambda_{L}(S, L) \\
& =\pi^{\prime}(L) \lambda(S, L)-\pi(L) \lambda(S, L) \lambda_{S}(L, L) \\
& =\lambda(S, L)\left[\pi^{\prime}(L)-V_{S}(L, L)\right] .
\end{aligned}
$$

(Formula (3.1.8) can also be derived using (3.8)). Since $\lambda(S, L)$ is positive, $V_{L}(S, L)=0$ if and only if

$$
V_{S}(L, L)=\pi^{\prime}(L) .
$$

Equation (3.1.8) shows explicitly that the optimal exercise boundary $\tilde{L}$ does not depend on the current stock price $S$. We note that (3.1.7), (3.1.8) and (3.1.9) are valid for payoff functions $\pi(\cdot)$ more general than (3.1.6).

Similarly, one can derive the formula

$$
W_{M}(S, M)=\mu(S, M)\left[\pi^{\prime}(M)-W_{S}(M, M)\right],
$$

where

$$
\mu(S, M)=E\left[e^{-\delta T_{M}} ; h^{*}\right] .
$$




\section{LOGARITHM OF THE STOCK PRICE AS A WIENER PROCESS}

The stochastic process with stationary and independent increments and sample paths which are both skip-free upwards and downwards (i.e., continuous) is the Wiener process. In this section we assume that $\{X(t)\}_{t \geq 0}$ is a Wiener process; this is the classical geometric Brownian motion model for stock-price movements (SAMUELSON, 1965; BLACK and SCHOLES, 1973). Let $\mu$ and $\sigma^{2}$ denote, respectively, the mean and variance of $\{X(t)\}$ per unit time. In terms of a stochastic differential equation, the assumption is

$$
\frac{d S(t)}{S(t)}=\left(\mu+\frac{\sigma^{2}}{2}\right) d t+\sigma d W(t), \quad t \geq 0,
$$

where $\{W(t)\}_{t \geq 0}$ denotes the standardized Wiener process.

Since

$$
M(z, t)=\exp \left[\left(\mu z+1 / 2 \sigma^{2} z^{2}\right) t\right]
$$

it follows from (2.5) that

$$
\ln [M(z, t ; h)]=\left[\left(\mu+h \sigma^{2}\right) z+1 / 2 \sigma^{2} z^{2}\right] t .
$$

This shows that the transformed process has modified mean per unit time $\mu+h \sigma^{2}$ and unchanged variance per unit time $\sigma^{2}$. From (2.8) we get

$$
\left(\mu+h^{*} \sigma^{2}\right)+1 / 2 \sigma^{2}=\delta-\rho
$$

Thus to evaluate a derivative security, we use a Wiener process with mean per unit time

$$
\mu+h^{*} \sigma^{2}=\delta-\rho-1 / 2 \sigma^{2}
$$

From (3.6) we obtain

$$
\left(\delta-\rho-1 / 2 \sigma^{2}\right) \theta+1 / 2 \sigma^{2} \theta^{2}=\delta,
$$

or

$$
\sigma^{2} \theta^{2}+\left(2 \delta-2 \rho-\sigma^{2}\right) \theta-2 \delta=0 .
$$

The roots of this quadratic equation are

$$
\theta_{0}=\frac{-\left(2 \delta-2 \rho-\sigma^{2}\right)-\sqrt{\left(2 \delta-2 \rho-\sigma^{2}\right)^{2}+8 \sigma^{2} \delta}}{2 \sigma^{2}}
$$

and

$$
\theta_{1}=\frac{-\left(2 \delta-2 \rho-\sigma^{2}\right)+\sqrt{\left(2 \delta-2 \rho-\sigma^{2}\right)^{2}+8 \sigma^{2} \delta}}{2 \sigma^{2}}
$$

Formula (4.5) should be attributed to MCKEAN (1965, Section 3) who studied the pricing of perpetual warrants; at that date of course he did not solve the problem in 
terms of the risk-neutral measure. With zero dividend yield $(\rho=0)$, formula (4.4) becomes

$$
\theta_{0}=\frac{-2 \delta}{\sigma^{2}}
$$

which was first given by Merton (1973, Section 8; 1990, Section 8.8), who evaluated the perpetual American put option by adopting McKEAN's (1965) technique. Discussions on pricing perpetual American options can also be found in the books by KARLIN and TAYLOR (1975, p. 365), INGERSOLL (1987, p. 375) and LAMBERTON and LAPEYRE (1991, p. 82), and in the recent articles by KARATZAS (1988, p. 59, e.g. 6.7), KIM (1990) and JACKA (1991, Proposition 2.3). (In formula (9) of KIM (1990), the denominator $1-\beta$ should be $\beta-1$ ).

In the finance literature, the formulas for pricing perpetual American options are usually derived as follows. Let $D$ denote the value of a derivative security. It follows from the hedging argument first given by BLACK and SHOLES (1973) that $D$ satisfies the partial differential equation

$$
1 / 2 \sigma^{2} S^{2} D_{S S}+(\delta-\rho) S D_{S}-\delta D+D_{t}=0,
$$

subject to the appropriate boundary conditions. In the case of a perpetual option, we have $D_{t}=0$ and (4.7) becomes a homogeneous, linear, second-order ordinary differential equation in $S$,

$$
1 / 2 \sigma^{2} S^{2} D_{S S}+(\delta-\rho) S D_{S}-\delta D=0 .
$$

The function $D=S^{\theta}$ is a solution of (4.8) if the number $\theta$ satisfies the quadratic equation,

$$
1 / 2 \sigma^{2} \theta(\theta-1)+(\delta-\rho) \theta-\delta=0,
$$

which is the same as (4.3). Then any solution of (4.8) is of the form

$$
D=c_{0} S^{\theta_{0}}+c_{1} S^{\theta_{1}},
$$

where $c_{0}$ and $c_{1}$ are independent of $S$.

In this paper we use the martingale approach and avoid differential equations. Additional insight for (4.10) is provided in the following; see (4.1.16) below.

\subsection{Perpetual contingent claims}

In this section we consider the pricing of perpetual contingent claims with $U$-shaped payoff functions such as

$$
\pi(x)=a_{1}\left(K_{1}-x\right)_{+}+a_{2}\left(x-K_{2}\right)_{+} .
$$

For $a_{1}=a_{2}=1$, the contingent claim may be called a perpetual American strangle if $K_{1}<K_{2}$, and called a perpetual American straddle if $K_{1}=K_{2}$. The assumption on $\{X(t)\}$ remains that it is a Wiener process. 
Let $S=S(0)$ denote the current stock price. We consider exercise strategies arising from stopping times of the form

$$
T_{L, M}=\inf \{t \mid S(t)=L \text { or } S(t)=M\},
$$

where $0 \leq L \leq S \leq M$. The value of the contingent claim according to such a strategy is

$$
V(S, L, M)=E\left[\pi\left(S\left(T_{L, M}\right)\right) e^{-\delta T_{L . M}} ; h^{*}\right] .
$$

Put

$$
\lambda(S, L, M)=E\left[I\left(S\left(T_{L, M}\right)=L\right) e^{-\partial T_{L . M}} ; h^{*}\right]
$$

and

$$
\mu(S, L, M)=E\left[I\left(S\left(T_{L, M}\right)=M\right) e^{-\delta T_{L, M}} ; h^{*}\right] .
$$

Then

$$
V(S, L, M)=\pi(L) \lambda(S, L, M)+\pi(M) \mu(S, L, M) .
$$

For $\theta=\theta_{0}$ and $\theta=\theta_{1}$ (the roots of equation (4.3)), the process $\left\{e^{-\partial t+\theta X(t)}\right\}$ is a bounded martingale (with respect to the risk-neutral measure) for $t \leq T_{L, M}$. Applying the optional sampling theorem to these two martingales yields the equations

$$
\lambda(S, L, M)\left(\frac{L}{S}\right)^{\theta_{0}}+\mu(S, L, M)\left(\frac{M}{S}\right)^{\theta_{0}}=1
$$

and

$$
\lambda(S, L, M)\left(\frac{L}{S}\right)^{\theta_{1}}+\mu(S, L, M)\left(\frac{M}{S}\right)^{\theta_{1}}=1,
$$

respectively, from which we obtain

$$
\lambda(S, L, M)=\frac{M^{\theta_{1}} S^{\theta_{0}}-M^{\theta_{01}} S^{\theta_{1}}}{M^{\theta_{1}} L^{\theta_{0}}-M^{\theta_{0}} L^{\theta_{i}}}
$$

and

$$
\mu(S, L, M)=\frac{S^{\theta_{1}} L^{\theta_{0}}-S^{\theta_{0}} L^{\theta_{1}}}{M^{\theta_{1}} L^{\theta_{0}}-M^{\theta_{0}} L^{\theta_{1}}} .
$$

Note that

$$
\lim _{M \rightarrow \infty} \lambda(S, L, M)=\left(\frac{S}{L}\right)^{\theta_{0}}=\left(\frac{L}{S}\right)^{-\theta_{0}},
$$


which confirms (3.7), and

$$
\lim _{L \downarrow \downarrow_{0}} \mu(S, L, M)=\left(\frac{S}{M}\right)^{\theta_{1}},
$$

which is (3.14).

The remaining problem is to optimize $V(S, L, M)$, considered as a function of the exercise boundaries $L$ and $M$. The first order conditions are

$$
V_{L}(S, \tilde{L}, \tilde{M})=0
$$

and

$$
V_{M}(S, \tilde{L}, \tilde{M})=0 .
$$

These conditions do not depend on $S$ (as long as $S$ is between $L$ and $M$ ). At first this seems surprising, but it follows immediately from the formulas

$$
V_{L}(S, L, M)=\lambda(S, L, M)\left[\pi^{\prime}(L)-V_{S}(L, L, M)\right]
$$

and

$$
V_{M}(S, L, M)=\mu(S, L, M)\left[\pi^{\prime}(M)-V_{S}(M, L, M)\right],
$$

which generalize (3.1.8) and (3.1.10), respectively. Thus the first order conditions become

$$
V_{S}(\tilde{L}, \tilde{L}, \tilde{M})=\pi^{\prime}(\tilde{L})
$$

and

$$
V_{S}(\tilde{M}, \tilde{L}, \tilde{M})=\pi^{\prime}(\tilde{M})
$$

which are the high contact conditions. The optimal exercise boundaries $\tilde{L}$ and $\tilde{M}$ are determined by solving (4.1.14) and (4.1.15) simultaneously. For $\tilde{L} \leq S \leq \tilde{M}$, the price of the perpetual contingent claim is

$$
\begin{aligned}
V(S, \tilde{L}, \tilde{M}) & =\pi(\tilde{L}) \lambda(S, \tilde{L}, \tilde{M})+\pi(\tilde{M}) \mu(S, \tilde{L}, \tilde{M}) \\
& =\left(\begin{array}{ll}
S^{\theta_{0}} & S^{\theta_{1}}
\end{array}\right)\left(\begin{array}{cc}
\tilde{L}^{\theta_{0}} & \tilde{L}^{\theta_{1}} \\
\tilde{M}^{\theta_{0}} & \tilde{M}^{\theta_{1}}
\end{array}\right)^{-1}\left(\begin{array}{c}
\pi(\tilde{L}) \\
\pi(\tilde{M})
\end{array}\right) .
\end{aligned}
$$

To prove (4.1.12), consider the identities

$$
\lambda(S, L, M)=\lambda(S, L+x, M) \lambda(L+x, L, M)
$$

and

$$
\mu(S, L, M)=\mu(S, L+x, M)+\lambda(S, L+x, M) \mu(L+x, L, M)
$$

where $0<x<S-L$. Differentiating these equations with respect to $x$ and setting $x=0$ yields

$$
0=\lambda_{L}(S, L, M)+\lambda(S, L, M) \lambda_{S}(L, L, M)
$$


and

$$
0=\mu_{L}(S, L, M)+\lambda(S, L, M) \lambda_{S}(L, L, M),
$$

respectively. Differentiating (4.1.5) with respect $L$ and applying (4.1.17) and (4.1.18), we have

$$
\begin{aligned}
V_{L}(S, L, M) & =\pi^{\prime}(L) \lambda(S, L, M)+\pi(L) \lambda_{L}(S, L, M)+\pi(M) \mu_{L}(S, L, M) \\
& =\lambda(S, L, M)\left[\pi^{\prime}(L)-\pi(L) \lambda_{S}(L, L, M)-\pi(M) \mu_{S}(L, L, M)\right] \\
& =\lambda(S, L, M)\left[\pi^{\prime}(L)-V_{S}(L, L, M)\right],
\end{aligned}
$$

which is (4.1.12). The proof of (4.1.13) is similar.

Remarks : For general payoff functions, there might be several disjoint optimal non-exercise intervals. For a matrix derivation of the results in this Section, see Section 5 of Gerber and SHIU (1994). There are closed-form formulas for deferred perpetual American call and put options; see Gerber and ShIU (1993b).

\subsection{Perpetual down-and-out option}

In this section we consider the pricing of a perpetual "down-and-out" American call option with exercise price $K$. The option contract becomes null and unexercisable, if the stock price declines to the knock-out price $L, L<K$. When this occurs, a rebate or refund of amount $R$ is given. For $M \geq K$, it follows from (4.1.5) that the value of the strategy to exercise the call option when the stock price increases to $M$ for the first time is

$$
V(S, L, M)=R \lambda(S, L, M)+(M-K) \mu(S, L, M), \quad L \leq S \leq M .
$$

Note that the lower exercise boundary $L$ is fixed, and the problem is to maximize $V$ as a function of the upper exercise boundary $M$.

We now consider the special case where the stock pays no dividends (hence $\theta_{1}=1$ and $\left.\theta_{0}=-2 \delta / \sigma^{2}\right)$. We shall show that the maximal value of (4.2.1) is obtained for $M \rightarrow \infty$ and that it is

$$
\begin{aligned}
V(S, L, \infty) & =S+(R-L)\left(\frac{L}{S}\right)^{-\theta_{0}} \\
& =S+(R-L)\left(\frac{L}{S}\right)^{2 \delta / \sigma^{2}} .
\end{aligned}
$$

This result can also be found in MerTon (1973, (57); 1990, (8.57)) and INGERSOLL (1987, p. 372, (39)).

For the proof we first observe that $\lambda(S, L, M)$ is an increasing function of $M$ and hence the first term on the right-hand side of (4.2.1) is bounded by

$$
R \lambda(S, L, \infty)=R\left(\frac{L}{S}\right)^{-\theta_{0}} .
$$


The second term on the right-hand side of (4.2.1) may be estimated as follows:

$$
\begin{aligned}
(M-K) \mu(S, L, M) & =(M-K) \frac{S L^{\theta_{0}}-S^{\theta_{0}} L}{M L^{\theta_{0}}-M^{\theta_{0}} L} \\
& =\frac{M-K}{M-\left(\frac{L}{M}\right)^{-\theta_{0}} L}\left[S-L\left(\frac{L}{S}\right)^{-\theta_{0}}\right] \\
& <S-L\left(\frac{L}{S}\right)^{-\theta_{0}} \\
& =\lim _{M \rightarrow \infty}(M-K) \mu(S, L, M) .
\end{aligned}
$$

\subsection{The Russian option}

Let $M$ be a number such that $M \geq S$. Let

$$
M(t)=\max \{M, \max [S(u) \mid 0 \leq u \leq t]\},
$$

which can be interpreted as the historical maximum of the stock prices at time $t$. Note that the pair $\{S(t), M(t) ; t \geq 0\}$ is a homogeneous Markov process. The term "Russian option" was coined by SHEPP and SHIRAYAEv (1993) to describe a perpetual American option whose payoff is $M(t)$, if it is exercised at time $t, t \geq 0$. That is, the holder of a Russian option has the privilege of receiving the historical maximum of the stock prices up till when he chooses to exercise the option. The price at time 0 of the option is the supremum, over all stopping times $T \geq 0$, of

$$
E\left[e^{-\delta T} M(T) ; h^{*}\right] \text {. }
$$

ShePp and ShIRYAEv (1993) show that there is a number $\tilde{k}$, which depends only on $\delta, \rho$ and $\sigma$, such that (if $S(0)>\tilde{k} M$ ) the optimal strategy is to exercise the option at the first time $t$ when

$$
S(t)=\tilde{k} M(t) .
$$

Here we shall show how $\tilde{k}$ can be determined in a very transparent fashion. Let $k$ be a number, $0<k<1$. For a current stock price $S=S(0)$ with $k M \leq S$, we consider the strategy to exercise the option at the stopping time

$$
T_{k}=\inf \{t \mid S(t)=k M(t)\} .
$$

The value of this strategy is denoted by $R(S, M ; k)$; we note that

$$
R(S, M ; k)=M R(S / M, 1 ; k) .
$$

From this and the definitions (4.1.3) and (4.1.4) it follows that

$$
\begin{aligned}
R(S, M ; k) & =M \lambda(S, k M, M)+R(M, M ; k) \mu(S, k M, M) \\
& =M[\lambda(S, k M, M)+R(1,1 ; k) \mu(S, k M, M)] .
\end{aligned}
$$


Applying (4.1.8) and (4.1.9), we obtain

$$
\begin{gathered}
R(S, M ; k)=M[\lambda(S / M, k, 1)+R(1,1 ; k) \mu(S / M, k, 1)] \\
=\frac{M}{k^{\theta_{0}}-k^{\theta_{1}}}\left\{\left[(S / M)^{\theta_{0}}-(S / M)^{\theta_{1}}\right]+R(1,1 ; k)\left[k^{\theta_{0}}(S / M)^{\theta_{1}}-k^{\theta_{1}}(S / M)^{\theta_{0}}\right]\right\},
\end{gathered}
$$

where $R(1,1 ; k)$ needs to be determined by the boundary condition at $S=M$. This condition can be derived by the following heuristic argument. If the current stock price $S$ is very close to $M$, we can be " almost sure" that the stock price will attain the level $M$ (and hence that the maximum will be increased) before the option is exercised. Thus, if $S$ is close to $M, R(S, M ; k)$ does not depend on the exact value of $M$,

$$
R_{M}(M, M ; k)=0 .
$$

From this and (4.3.6) we get the condition

$$
\frac{1}{k^{\theta_{0}}-k^{\theta_{1}}}\left\{\left[\left(1-\theta_{0}\right)-\left(1-\theta_{1}\right)\right]+R(1,1 ; k)\left[k^{\theta_{0}}\left(1-\theta_{1}\right)-k^{\theta_{1}}\left(1-\theta_{0}\right)\right]\right\}=0,
$$

which yields

$$
R(1,1 ; k)=\frac{\theta_{1}-\theta_{0}}{\left(1-\theta_{0}\right) k^{\theta_{1}}-\left(1-\theta_{1}\right) k^{\theta_{0}}} .
$$

We substitute this expression into formula (4.3.6) and obtain after simplification the result that

$$
R(S, M ; k)=M \frac{\left(1-\theta_{0}\right)(S / M)^{\theta_{1}}+\left(\theta_{1}-1\right)(S / M)^{\theta_{11}}}{\left(1-\theta_{0}\right) k^{\theta_{1}}+\left(\theta_{1}-1\right) k^{\theta_{0}}} .
$$

Now it is clear that the optimal value of $k$ is the one that minimizes the denominator, whose derivative is

$$
\left(1-\theta_{0}\right) \theta_{1} k^{\theta_{1}-1}+\left(\theta_{1}-1\right) \theta_{0} k^{\theta_{0}-1} .
$$

Hence the optimal value is

$$
\tilde{k}=\left(\frac{\theta_{0}\left(1-\theta_{1}\right)}{\theta_{1}\left(1-\theta_{0}\right)}\right)^{1 /\left(\theta_{1}-\theta_{0}\right)}
$$

and the price of the Russian option is

$$
\left\{\begin{array}{lll}
R(S, M ; \tilde{k}) & \text { if } & \tilde{k} \mathrm{M} \leq \mathrm{S} \leq \mathrm{M} \\
M & \text { if } & S \leq \tilde{k} \mathrm{M}
\end{array} .\right.
$$

Formulas (4.3.10) and (4.3.11) are equivalent to (2.3) and (2.4) of SHEPP and SHIRYAEV (1993). 


\section{ACKNOWLEDGEMENTS}

The authors thank FrançOIS DUFRESNE for pointing out the paper by SHEPP and ShiryaeV (1993) and the anonymous referees for their comments. Elias SHIU gratefully acknowledges the support from the Principal Financial Group.

\section{REFERENCES}

BARONE-ADESI, G. and Whaiey, R.E. (1987) Efficient analytic approximation of American option values. Journal of Finance 42, 301-320.

Bhattacharya, S. and Constantinides, G. (ed.) (1989) Theory of Valuation: Frontier of Modern Financial Theory Volume 1. Rowman and LitTlefiel.d, Totowa, N.J.

BlaCk, F. and SCHOLES, M. (1973) The pricing of options and corporate liabilities. Journal of Political Economy 81, 637-659.

Bensoussan, A. (1984) On the theory of option pricing. Acta Applicandae Mathematicae 2, 139-158.

Breiman, L. (1968) Probability. Addison-Wesley, Menlo Park, California, Reprinted by the Society of Industrial and Applied Mathematics, Philadelphia, 1992.

BREKKE, K. A. and ØKSENDAL, B. (1991) The high contact principle as a sufficiency condition for optimal stopping. In LUND and ØKSENDAL. (1991), 187-208.

CARR, P., JARROW, R. and MYNENl, R. (1992) Alternative characterizations of American put options. Mathematical Finance 2, 87-106.

Chesney, M., Elllott, R. J. and Gibson, R. (1993) Analytical solutions for the pricing of American bond and yield options. Mathematical Finance 3, 277-294.

COOTNER, P. H. (ed.) (1967) The Random Character of Stock Market Prices, revised edition. M.I.T. Press, Cambridge, Massachusetts.

Cox, J.C. and Ross, S.A. (1976) The valuation of options for alternative stochastic processes. Journal of Financial Economics 3, 145-166.

Delbaen, F. and Hafzendonck, J. (1989) A martingale approach to premium calculation principles in an arbitrage free market. Insurance: Mathematics and Economics 8, 269-277.

Dufresne, F., Gerber H.U. and ShiU, E.S.W. (1991) Risk theory with the gamma process. ASTIN Bulletin 21, 177-192.

Dybvig, P. H. and Ross, S. A. (1987) Arbitrage. In The New Palgrave: A Dictionary of Economics Vol. 1, edited by J. Eatwill., M. Milgate and P. Newman, Macmillan, London, 100-106. Reprinted in The New Palgrave: Finance, edited by J. Eatwell, M. Milgate and P. Newman, W. W. Norton, New York (1989), 57-71.

ESSCHER, F. (1932) On the probability function in the collective theory of risk. Skandinavisk Aktuarietidskrift 15, 175-195.

Gerber, H. U. and Shiu, E.S.W. (1993a) Option pricing by Esscher transforms. Proceedings of the 24th ASTIN Colloquium at Cambridge University Volume 2, 305-344. Also to appear in the Transactions of the Society of Actuaries 46, (1994).

Gerber, H. U. and Shul, E. S.W. (1993b) Discussion on Tilley (1993). Transactions of the Society of Actuaries 45.

Gerber, H.U. and ShiU, E.S.W. (1994) From perpetual strangles to Russian options. Insurance: Mathematics and Economics.

Goldman, M. B., Sosin, H. B. and Gatto, M. A. (1979) Path dependent options: "Buy at the low, sell at the high". Journal of Finance 34, 1111-1127.

Harrison, J.M. and KrePS, D.M. (1979) Martingales and arbitrage in multiperiod securities markets. Journal of Economic Theory 20, 381-408.

Harrison, J.M. and Pliska, S. (1981) Martingales and stochastic integrals in the theory of continuous trading. Stochastic Processes and Their Applications 11, 215-260.

HeSTON, S. L. (1993) Invisible parameters in option prices. Journal of Finance 48, 933-947.

Hul.., J. and White, A. (1993) Efficient procedures for valuing European and American path-dependent options. Journal of Derivatives 1, 21-31.

Ingersoll, J.E., Jr. (1987) Theory of Financial Decision Making. Rowan and Littlefield, Totowa, N.J.

JACKA, S. D. (1991) Optimal stopping and the American put. Mathematical Finance 1 (2), 1-14. 
Jaillet, P., Lamberton, D. and LAPEyre, B. (1990) Variational inequalities and the pricing of American options. Acta Applicandae Mathematicae 21, 263-289.

Karatzas, I. (1988) On the pricing of American options. Applied Mathematics and Optimization 17, $37-60$.

Karlin, S. and Taylor, H. (1975) A First Course in Stochastic Processes (2nd edn). Academic Press, New York.

Karlin, S. and Taylor, H. (1981) A Second Course in Stochastic Processes. Academic Press, New York.

KıM, I. J. (1990) The analytic valuation of American options. Review of Financial Studies 3, 545-572.

LAMBERTON, D. (1993) Convergence of the critical price in the approximation of American options. Mathematical Finance 3, 179-190.

LAMBERTON, D. and LAPEYRE, B. (1991) Introduction au calcul stochastique appliqué à la finance. Mathématiques \& Applications \#9, Société de Mathématiques Appliquées et Industrielles; distributed by Ellipses, Paris, France.

Lund, D. and $\emptyset_{\mathrm{KSENDAL}}$, B. (ed.) (1991) Stochastic Models and Option Values: Applications to Resources, Environment and Investment Problems. North-Holland, Amsterdam.

MacMillan, L.W. (1986) Analytic approximation for the American put. Advances in Futures and Options Research 1 (A), 119-139.

McKeAN, H.P. (1965) A free boundary problem for the heat equation arising from a problem in mathematial economics: An appendix to SAMUELSON (1965). Industrial Management Review 6 (2), 32-39. Reprinted in CoOTNER (1967), 525-532.

MERTON, R.C. (1973) Theory of rational option pricing. Bell Journal of Economics and Management Science 4, 141-183. Reprinted in BhatTacharya and Constantinides (1989), 229-271. and as Chapter 8 in MERTON (1990), 225-308.

Merton, R. C. (1990) Continuous-Time Finance. Basil Blackwell, Oxford.

Myneni, R. (1992) The pricing of the American option. Annals of Applied Probability 2, 1-23.

OMBERG, E. (1987) The valuation of American put options with exponential exercise polices. Advances in Futures and Options Research 2, 117-142.

SAMUElson, P. A. (1965) Rational theory of warrant pricing. Industrial Management Review 6 (2), 13-32. Reprinted in COOTNER (1967), 506-525.

SCHACHERMAYER, W. (1992) A Hilbert space proof of the fundamental theorem of asset pricing in finite discrete time. Insurance: Mathematics and Economics 11, 249-257.

Shepp, L. and Shiryaev, A. N. (1993) The Russian option : Reduced regret. Annals of Applied Probability 3, 631-640.

Shirayayev, A. N. (1978) Optimal Stopping Rules. Springer-Verlag, New York.

SMith, C. W., Jr. (1976) Option pricing: A review. Journal of Financial Economics 3, 3-51.

TiLleY, J.A. (1993) Valuing American options in a path simulation model. Transactions of the Society of Actuaries 45.

\section{APPENDIX}

\section{A1. Semi-continuous sample paths}

In the rest of this paper, we consider the assumption that the sample paths of $\{S(t)\}$, or equivalently, those of $\{X(t)\}$, are skip-free downwards. (This assumption was used in deriving (3.10)). Then the following decomposition holds:

(A.1.1)

$$
X(t)=Y(t)+v^{2} W(t)-c t, \quad t \geq 0 .
$$

Here, $\{Y(t)\}$ is either a compound Poisson process with positive increments or the limit of such processes; $\{W(t)\}$ is an independent standardized Wiener process (with zero drift and unit variance per unit time); the last term, $c t$, represents a deterministic drift. The cumulant generating function of the random variable $X(t)$ is of the form

$$
\ln [M(z, t)]=t\left\{\int_{0}^{\infty}\left(e^{z x}-1\right)[-d Q(x)]+v^{2} z^{2} / 2-c z\right\},
$$


where $Q(x)$ is some nonnegative and nonincreasing function with $Q(\infty)=0$. Note that, for each positive number $\varepsilon$, the integral

$$
\int_{\varepsilon}^{x}\left(e^{\tau x}-1\right)[-d Q(x)]
$$

as a function in $z$, is the cumulant generating function of a compound Poisson distribution with Poisson parameter

$$
\lambda(\varepsilon)=Q(\varepsilon)
$$

and jump amount distribution

$$
P(x ; \varepsilon)=\frac{Q(\varepsilon)-Q(x)}{Q(\varepsilon)}, \quad x \geq \varepsilon .
$$

For notational simplicity, we assume that

$$
-d Q(x)=q(x) d x
$$

for some nonnegative function $q(x)$. Let $\mu$ and $\sigma^{2}$ denote, respectively, the mean and variance of $\{X(t)\}$ per unit time. Then

$$
\begin{aligned}
& \mu t=E[X(t)]=\left[\int_{0}^{\infty} x q(x) d x-c\right] t \\
& \sigma^{2} t=\operatorname{Var}[X(t)]=\left[\int_{0}^{\infty} x^{2} q(x) d x+v^{2}\right] t,
\end{aligned}
$$

and

$$
E\left[(X(t)-\mu t)^{3}\right]=t \int_{0}^{\infty} x^{3} q(x) d x
$$

In general, for $n \geq 3$, the $n$-th cumulant of $X(t)$ is given by

$$
t \int_{0}^{\infty} x^{n} q(x) d x
$$

If follows from (2.5) and (A.1.2) that

(A.1.6) $\ln [M(z, t ; h)]=\ln [M(z+h, t)]-\ln [M(h, t)]$

$$
=t\left\{\int_{0}^{x}\left(e^{z x}-1\right) e^{h x} q(x) d x+v^{2} z^{2} / 2-\left(c-v^{2} h\right) z\right\} .
$$


Thus the Esscher transform (parameter $h$ ) of a process defined by (A.1.1) is of the same type, with the following modifications:
$q(x) \rightarrow e^{h x} q(x)$,
(A.1.8)
$v^{2} \rightarrow v^{2} \quad$ (unchanged),
(A.1.9)
$c \quad \rightarrow c-v^{2} h$.

Furthermore, it follows from (A.1.6) that (2.8) and (3.6) can be written as

$$
\int_{0}^{\infty}\left(e^{x}-1\right) e^{h^{*} x} q(x) d x+\nu^{2} h^{*}=c+\delta-\rho-\frac{v^{2}}{2}
$$

and

$$
\int_{0}^{\infty}\left(e^{\theta x}-1\right) e^{h^{*} x} q(x) d x+\frac{v^{2} \theta^{2}}{2}-\left(c-v^{2} h^{*}\right) \theta=\delta,
$$

respectively.

\section{A2. A particular family}

For the model defined by (2.1) and (A.1.1), we now assume that $v=0$, i.e.,

$$
S(t)=S(0) e^{Y(t)-t t},
$$

and that

$$
q(x)=a x^{\alpha-1} e^{-b x}, \quad x>0,
$$

where $a>0, \alpha>-1$, and $b>0$ are three parameters. In the context of risk theory, Dufresne, Gerber and SHIU (1991) have considered such a $q(x)$ function.

According to (A.1.7), for $h<b$, the Esscher transform of a process defined by (A.2.1) is a member of the same family, with $b$ replaced by

$$
b(h)=b-h .
$$

The moment generating function of $Y(t)$ is

$$
\begin{aligned}
\exp \left[t \int_{0}^{\infty}\left(e^{z x}-1\right) q(x) d x\right] & =\exp \left[a t \int_{0}^{x}\left(e^{z x}-1\right) x^{\alpha-1} e^{-b x} d x\right] \\
& = \begin{cases}\left(\frac{b}{b-z}\right)^{a t} & \text { if } \alpha=0 \\
\exp \left(\frac{a \Gamma(\alpha)}{b^{\alpha}}\left[\left(\frac{b}{b-z}\right)^{\alpha}-1\right] t\right) & \text { if } \alpha \neq 0\end{cases}
\end{aligned}
$$


Thus, for $\alpha=0,\{Y(t)\}_{t \geq 0}$ is a gamma process; for $\alpha>0$, it is a compound Poisson process with Poisson parameter

$$
\lambda(a, \alpha, b)=\frac{a \Gamma(\alpha)}{b^{\alpha}},
$$

and gamma jump density

$$
p(x ; \alpha, b)=\frac{b^{\alpha}}{\Gamma(\alpha)} x^{\alpha-1} e^{-b x}, \quad x>0 .
$$

For $-1<\alpha<0$, the most prominent case is $\alpha=-1 / 2$, where $\{Y(t)\}_{t \geq 0}$ is an inverse Gaussian process and the density function of $Y(t)$ is

$$
\frac{a t}{x^{3 / 2}} \exp \left[\frac{-(\sqrt{b} x-\sqrt{\pi} a t)^{2}}{x}\right], \quad x>0 .
$$

The condition for

$$
b^{*}=b\left(h^{*}\right)=b-h^{*}
$$

becomes

$$
\frac{b^{*}}{b^{*}-1}=e^{\frac{c+\partial-\rho}{a}} \quad \text { if } \quad \alpha=0
$$

and

$$
\frac{1}{\left(b^{*}-1\right)^{\alpha}}-\frac{1}{b^{* \alpha}}=\frac{c+\delta-\rho}{a \Gamma(\alpha)} \quad \text { if } \quad \alpha \neq 0 .
$$

Solving (A.2.4) yields

$$
b^{*}=\frac{1}{1-e^{-(c+\delta-\rho) / a}},
$$

which, with $\rho=0$, is formula (3.1.7) in GERBER and SHIU (1993a). In general, equation (A.2.5) does not yield a closed-form solution for $b^{*}$. However, if $\alpha=1$ (exponential jump amounts), one finds

$$
b^{*}=\frac{1+\sqrt{1+\frac{4 a}{c+\delta-\rho}}}{2} .
$$

A discussion of the case where $\alpha=-1 / 2$ can be found in GERBER and SHIU (1993a).

For each fixed $\alpha$, we might determine the parameters, $a, b$ and $c$, by the method of moments. Thus we assume that we know $\mu, \sigma$ and the third central moment of $X(1)$, which we write as $\gamma \sigma^{3}$ ( $\gamma$ being the coefficient of skewness). Matching the 
first three moments (by means of formulas (A.1.3), (A.1.4) and (A.1.5)) yields the equations :

$$
\begin{gathered}
\mu=\int_{0}^{\infty} x q(x) d x-c=\frac{a \Gamma(\alpha+1)}{b^{\alpha+1}}-c, \\
\sigma^{2}=\int_{0}^{\infty} x^{2} q(x) d x=\frac{a \Gamma(\alpha+2)}{b^{\alpha+2}},
\end{gathered}
$$

and

$$
\gamma \sigma^{3}=\int_{0}^{x} x^{3} q(x) d x=\frac{a \Gamma(\alpha+3)}{b^{\alpha+3}} .
$$

From these equations we obtain

$$
b=\frac{\alpha+2}{\gamma \sigma}
$$

(to be replaced by $b^{*}$ for the evaluation of a derivative security),

$$
a=\frac{(\alpha+2)^{\alpha+2}}{\Gamma(\alpha+2) \gamma^{\alpha+2} \sigma^{\alpha}},
$$

and

$$
c=\frac{\alpha+2}{\alpha+1} \frac{\sigma}{\gamma}-\mu .
$$

These formulas generalize (and explain!) the formulas in Sections V.2 and V.3 of Gerber and SHIU (1993a). We note that HESTON (1993) has independently introduced the gamma process for modeling stock-price movements; his formula (10a) is the same as formula (4.1.7) of GERBER and ShIU (1993a).

\section{A3. Formulas for the negative root}

With the assumptions $v=0$ and

$$
q(x)=a x^{\alpha-1} e^{-b x}, \quad x>0,
$$

equation (A.1.11) becomes

$$
a \int_{0}^{\infty}\left(e^{\theta x}-1\right) e^{h^{x} x} x^{\alpha-1} e^{-b x} d x-c \theta=\delta,
$$

or

(A.3.1)

$$
\int_{0}^{\infty}\left(e^{\theta x}-1\right) x^{\alpha-1} e^{-b^{\prime} x} d x=\frac{\delta+c \theta}{a}
$$


The value of the integral in the left-hand side of (A.3.1.) can be read off from (A.2.3).

If $\alpha=0$, then (A.3.1) becomes

$$
\frac{b^{*}}{b^{*}-\theta}=e^{\frac{\partial+c \theta}{a}} .
$$

Substituting $b^{*}$ in (A.3.2) with formula (A.2.6) yields

$$
e^{-c \cdot \theta / a}+\theta\left[e^{\delta / a}-e^{-(c-\rho) / a}\right]=e^{\delta / a} \text {. }
$$

By (A.2.8) and (A.2.9),

$$
\frac{1}{a}=\frac{\gamma^{2}}{4}
$$

and

$$
\frac{c}{a}=\frac{\gamma}{2}\left(\sigma-\frac{\mu \gamma}{2}\right) .
$$

For example, assume that $\delta=0.1, \rho=0, \mu=0.1, \sigma=0.2$ and $\gamma=1$. Then (A.3.3) becomes

$$
e^{-3 \theta / 40}+\theta\left[e^{1 / 40}-e^{-3 / 40}\right]=e^{1 / 40},
$$

from which we obtain

$$
\theta_{0}=-7.559609675 \text {. }
$$

Note that, in the Wiener process model (with $\delta=0.1$ and $\sigma=0.2$ ), $\theta_{0}=-5$ by formula (4.6).

If $\alpha \neq 0$ and $\alpha>-1$, then (A.3.1) becomes

$$
\frac{1}{\left(b^{*}-\theta\right)^{\alpha}}-\frac{1}{b^{* \alpha}}=\frac{\delta+c \theta}{a \Gamma(\alpha)},
$$

where $b^{*}$ is defined by (A.2.5). In the special case where $\alpha=1,(\mathrm{~A} .3 .4)$ simplifies as

$$
\frac{1}{b^{*}-\theta}=\frac{1}{b^{*}}=\frac{\delta+c \theta}{a},
$$

which is a quadratic equation in $\theta$, where $b^{*}$ is given by (A.2.7),

$$
a=\frac{27}{2 \gamma^{3} \sigma}
$$

and

$$
c=\frac{3}{2} \frac{\sigma}{\gamma}-\mu
$$


Now, consider the zero dividend case $(\rho=0)$, then the positive root of (A.3.5) is $\theta_{1}=1$, and the negative root is

$$
\theta_{0}=-\delta b^{*} / c
$$

using the same numerical values as above, $\delta=0.1, \mu=0.1, \sigma=0.2$ and $\gamma=1$, we obtain

$$
b^{*}=\frac{1+\sqrt{901}}{2}
$$

and

$$
\theta_{0}=-7.75416551
$$

Hans U. Gerber

Ecole des hautes études commerciales,

Université de Lausanne,

CH-1015 Lausanne, Switzerland.

Elias S. W. ShIU

Department of Statistics and Actuarial Science,

The University of Iowa,

Iowa City, lowa 52242-1419, U.S.A. 